\title{
Preparation of Magnetorheological Fluid Using Stabilizing Additives
}

\author{
Aya KAIDE ${ }^{1}$, Makoto KANDA ${ }^{2}$, Hiroshi TOCHIGI ${ }^{2}$, and Takashi SAEKI ${ }^{*}$ \\ ${ }^{1}$ Department of Environmental Science and Engineering, Graduate School of Science and Technology for Innovation, Yamaguchi \\ University, 2-16-1, Tokiwadai, Ube-shi, Yamaguchi 755-8611, Japan \\ ${ }^{2}$ Cosmo Oil Lubricants Co., Ltd., 1134-2, Gongendo, Satte-shi, Saitama 340-0193, Japan
}

\begin{abstract}
In this study, we examined three kinds of stabilizing additives to prepare stable MRF with good fluidity. We selected three stabilizing additives; a styrene isoprene block copolymer, a hydrophobic fumed silica, and an organogelator (CMOL SB03; PMDA-2C 8 /oleyl). We measured the rheological properties both for the dissolutions of the stabilizing additives in the base oil and MRFs prepared with the additives by using a rheometer; MCR302 (Anton Paar Corp.). From the experimental results, the viscosity of styrene isoprene block copolymer dissolution increased with concentration showing non-Newtonian properties. We selected a suitable concentration as $5 \mathrm{wt} \%$ to satisfied good fluidity without forming gel. Since the viscosities of $7 \mathrm{wt} \%$ fumed silica and $1 \mathrm{wt} \%$ CMOL SB 03 dissolutions in the base oil consequently showed similar viscosity of the $5 w t \%$ copolymer, MRFs using such conditions were prepared. From the results of the stability test, the addition of the copolymer was effective to satisfy the stability of the MRF. We also found that the addition of such stabilizing additives were effective to increase the magnetic property of MRFs.
\end{abstract}

\section{Introduction}

A magnetorheological fluid (MRF) is an oil slurry with magnetic particles. MRF attracts much attention as a functional fluid, of which viscosity can be controlled by magnetic field (Rabinow, 1948). The fluidity of MRF relates the viscosity of a base oil and the size and the concentration of particles. Also, we the preparation conditions are not suitable, particle sedimentation and/or oil separation often occur due to the density difference and familiarity of the particles and the base oil. Using smaller particles can increase the stability of MRF. Surface coating of magnetic particles is also effective on the stability (Fang et al., 2012); however, the magnetic characteristic decreases for both methods. The addition of nanoparticles (Park et al., 2009) and increasing the viscosity of the base oil (Kim et al., 2012) are effective to increase not only the stability, but also the magnetic characteristics. However, the viscosity of MRF increases, consequently the slurry loses its fluidity.

The objective of this study was to increase the sedimentation stability of MRF by using suitable stabilizing additives. We selected three kinds of additives and considered the mechanism of stabilization with the connection of the network structure build by the additives and interaction with the magnetic particles. We also examined the effect of such additives on the magneto-rheological characteristics of MRF.

\section{Experimental}

\subsection{Materials and preparation}

The base oil used in this study was a highly refined paraffinic mineral oil (mineral oil-P4, kinematic viscosity at $40^{\circ} \mathrm{C} 2.0 \times 10^{-5} \mathrm{~m}^{2} / \mathrm{s}$, viscosity index 122 ). The magnetic particles were carbonyl iron powder (CS, BASF) having a density of $7860 \mathrm{~kg} / \mathrm{m}^{3}$. The 10,50 , and $90 \%$ pass diameters of the powder were $2.8,5.6,12.3$ $\mu \mathrm{m}$, respectively. We examined the following three stabilizing additives.

1) Styrene isoprene block copolymer

Average molecular weight: 284,000 .

2) Hydrophobic fumed silica

Aaverage particle size: $16 \mathrm{~nm}$

3) Organogelatore; CMOL SB03 (Daicel Corp.)

$N, N, N$ ", $N$ "'-1,2,4,5-tetra alkyl/alkenyl pyromellitamide (PMDA-R) with two 2-ethyl hexyls and two oleyls as branch alkyl groups (Sakanishi et al., 2015)

Each stabilizing additive was stirred into the base oil at $80^{\circ} \mathrm{C}$ for $5 \mathrm{~h}-2$ days until well mixed and/or dissolution. We continued to stir while lowering the room temperature over a period of $1 \mathrm{~h}$, then left the solutions to rest overnight at room temperature. Then, each sample was mixed with the carbonyl iron powder to prepare MRF. The solid concentration was set at $75 \mathrm{wt} \%$ (24.3

\footnotetext{
Corresponding author: kaide@yamaguchi-u.ac.jp
} 
vol $\%$ ). We added $1.0 \mathrm{wt} \%$ oleic acid as a dispersant, together with $0.5 \mathrm{wt} \%$ high molecular weight phenolic antioxidant and $0.5 \mathrm{wt} \%$ alkenyl succinimide as an ashless detergent dispersant. They were mixed using a bladeless rotation/revolution mixing apparatus (Awatori Rentaro, ARE-250, Thinky Corp.) at $2000 \mathrm{rpm}$ for $5 \mathrm{~min}$ to obtain MSF samples.

\subsection{Rheological measurement}

The rheological properties of the stabilizer samples in the oil were measured using a strain-control type cone and plate rheometers (MCR 302, Anton Paar). Steady flow characteristics were measured for shear rate in the range from $0.01-1000 \mathrm{~s}^{-1}$. The measurement temperature was set at $25 \pm 0.5^{\circ} \mathrm{C}$ with a Peltier control system. In casting a sample on the rheometer plate, we left it for more than 5 min until the temperature arrived at the set point.

\subsection{Stability test}

Each MRF was poured into a $10 \mathrm{~mL}$ graduated cylinder and left at rest (Figure 1). After three weeks (504 h), the height of an oil layer formed in the upper of the cylinder was meaured to calculate the oil separation ratio, $\varphi$, defined as the following :

$\varphi=v / V$

(1),

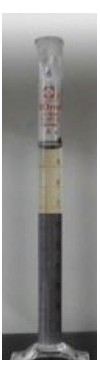

where $v$ and $V$ are the volume of the oil layer and total volume of the oil

Figure 1. Stability test

of the MRF. The measurement temperature was set at $20 \pm 0.5^{\circ} \mathrm{C}$ with a Peltier control system.

\section{Results and Discussions}

Figure 2 shows the steady flow characteristics of styrene isoprene block copolymer dissolutions with different concentrations. $1 \mathrm{wt} \%$ dissolution showed the constant viscosity with shear rate (Newtonian fluid). The dissolutions of $2-4 \%$ showed shear thinning properties gently depending on the shear rate of more than $10 \mathrm{~s}^{-1}$. The addition of more than $5 \%$ caused remarkable shear thinning properties over the whole range of the shear rate.

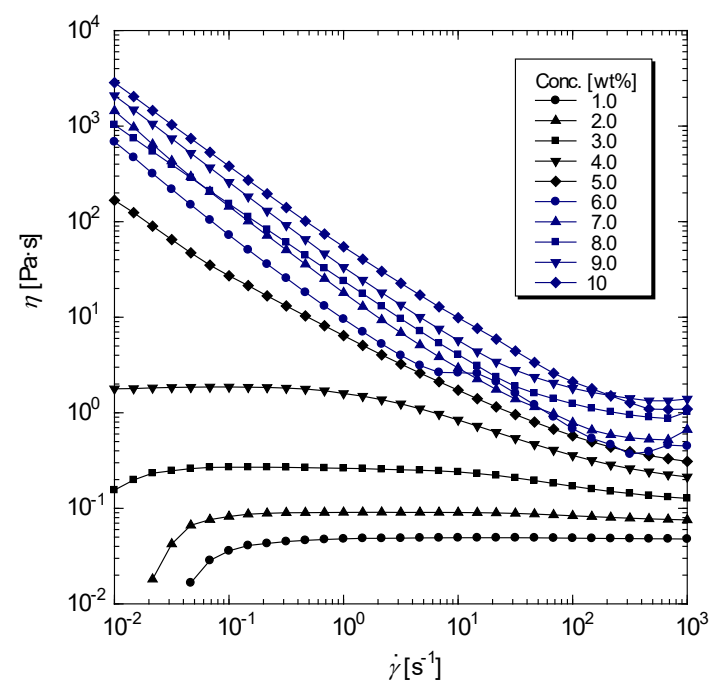

Figure 2. Steady flow characteristics of styrene isoprene block copolymer dissolution with different concentrations

Figure 3 shows the dynamic viscoelastic moduli for styrene isoprene block copolymer dissolution (1-5\%).

The morphology of the structure of the styrene isoprene block copolymer was observed using a TEM (JEM-140; JEOL Co., Tokyo, Japan) with an accelerating voltage of $100 \mathrm{kV}$. In the preprocessing, the sample was dissolved in the base oil, and sample was attached to a support film grid and stained with osmium tetraoxide $2 \%$ aqueous solution for $4 \mathrm{~h}$ to achieve osmium coating on the surface of self-assembled structures.

\subsection{Magneto-rheological characteristic}

The magneto-rheological characteristics of MRFs were measured using a strain-control type rheometer (MCR 101, Anton Paar Corp.) with a parallel plates arrangement with the distance of $0.5 \mathrm{~mm}$. The diameter of the plate was $19.956 \mathrm{~mm}$. $0.2 \mathrm{~mL}$ MRF sample was catsted to a Magneto-rheological device cell (MRD170/1T) with TwinGap ${ }^{\mathrm{TM}}$ geometry (TG/MRD) having the gap of $0.3 \mathrm{~mm}$ each in order to apply a constant magnetic flux density, $B$. The shear stress, $\tau$, was meaured with varying magnetic flux density at constant shear rate of $100 \mathrm{~s}^{-1}$. Thus obtained $B-\tau$ chart was used to evaluate magneto-rheological characteristic

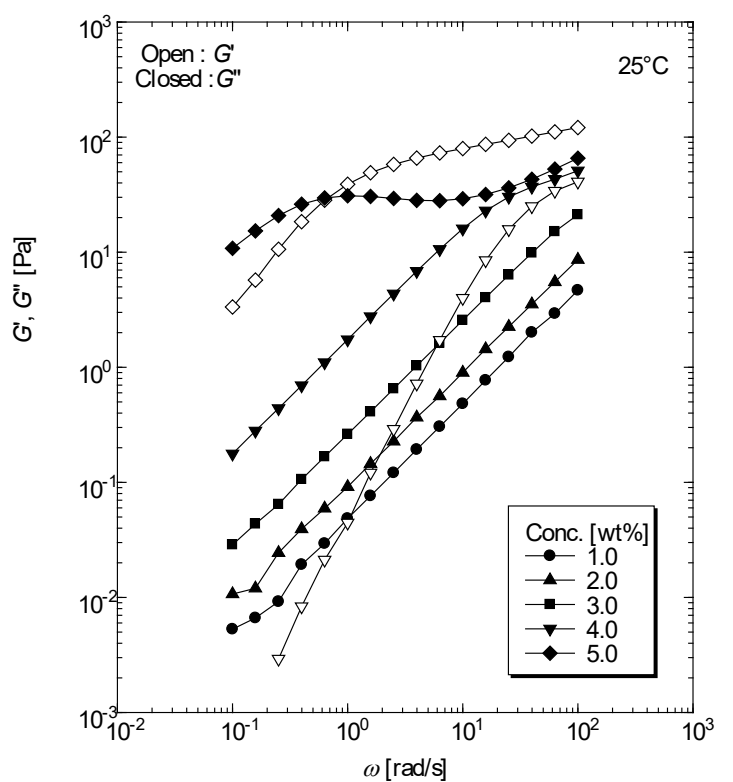

Figure 3. Dynamic viscoelastic moduli for styrene isoprene block copolymer dissolution (1-5 wt\%) 
Since the values of loss modulus, G", for $1-3 \mathrm{wt} \%$ were proportional to $\omega$, we found that these samples were viscous fluids without the elastic properties. According to the data of $4 \mathrm{wt} \%, G$ " was also proportional to $\omega$, while storage modulus, $G^{\prime}$, indicating the elasticity was proportional to $\omega^{2}$. Furthermore, when we focused on the data of $5 \mathrm{wt} \%$, since $G$ " and $G$ ' were proportional to $\omega$ and $\omega^{2}$, respectively, at lower frequency, the sample was a typical viscoelastic fluid. The value of $\omega$ at the cross point of $G^{\prime}$ and $G$ "' was $0.63 \mathrm{rad} / \mathrm{s}$. The expression of the viscoelastic properties may cause by the interaction among the stabilizer molecules at higher concentrations; however, the dissolutions did not form gel structure in this concentration range.

Figure 4 shows the results of higher concentration $(6-10 \mathrm{wt} \%)$ of the same stabilizing addtives. Since $G$, exceeded $G$ " independently of $\omega$, we found that the dissolutions were gel states and $G$ ' gradually increased with the increase of the copolymer concentration. Since the dissolutions became gels by adding more than $6 \mathrm{wt} \%$, we selected a suitable concentration as $5 \mathrm{wt} \%$ to satisfied good fluidity of MRF.

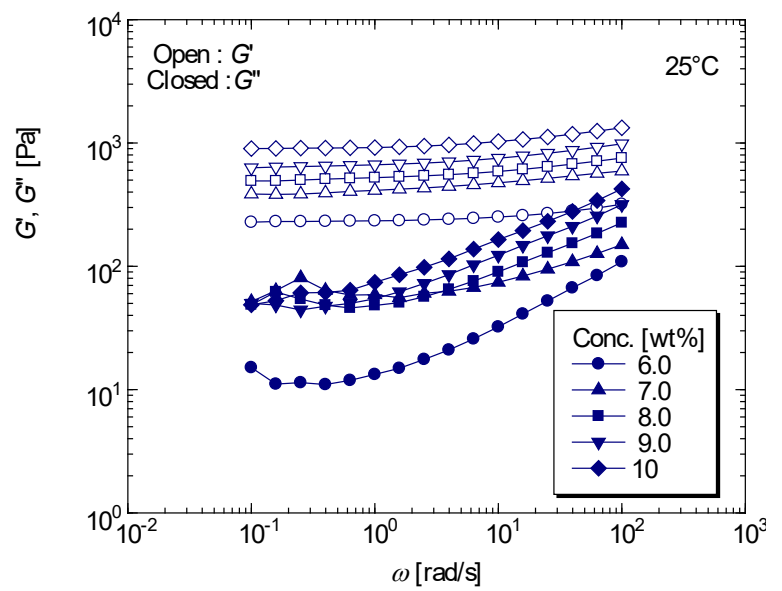

Figure 4. Dynamic viscoelastic moduli for styrene isoprene block copolymer dissolution (6-10 wt\%)

Figure 5 shows the steady flow characteristics of MRFs. MRF prepared only the iron powder and the base oil $(\nabla)$ showed a shear thinning property for the shear rate more than $1 \mathrm{~s}^{-1}$. The viscosity at $1000 \mathrm{~s}^{-1}$ decreased one order of magnitude compared with that at $1 \mathrm{~s}^{-1}$. With the addition of $1 \mathrm{wt} \%$ oleic acid as a dispersant $(\boldsymbol{\nabla})$, the viscosity of the MRF decreased. With the addition of 5 $\mathrm{wt} \%$ styrene isoprene block copolymer to the MRF (containing $1 \mathrm{wt} \%$ oleic acid), the viscosity increased tenfold showing significant shear thinning property. The addition of $7 \mathrm{wt} \%$ fumed silica and $1 \mathrm{wt} \%$ CMOL SB 03 consequently showed similar viscosity increasing abilities.

Figure 6 shows stability test of MRFs, of which the horizontal axis shows the time after left at rest. MRF prepared with the iron powder and the base oil (without oleic acid) showed oil separation ratio of more than $30 \%$ after one day and finally reached to $34.8 \%(\nabla)$. In contrast, the oil separation ratio increased as $60.7 \%$ with the addition of oleic acid $(\boldsymbol{\nabla})$. Since the added oleic acid worked as a surfactant, coagulated and/or agglomerated iron particles dispersed in the oil and settled for several days. As a result, the oil separation layer was formed. The settling layer of the particles was more rigid than that formed in the MRF without oleic acid. We have defined a criterion of oil separation ratio as $3 \%$ for quality control of MRF products. Since the oil separation of the copolymer fainally showed $2.4 \%(\boldsymbol{\Delta})$, we judged that the additive was effective to prevent the oil separation. Although the addition of $7 \mathrm{wt} \%$ fumed silica (•) was also effective to decrease the oil separation ratio (the ratio was $14.4 \%$ ), the ability was not enough for the criterion. In contrast, MRF using CMOL SB03 showed significant separation of $54 \%$ after $50 \mathrm{~h}$ (घ, finally became $54.2 \%$ ).

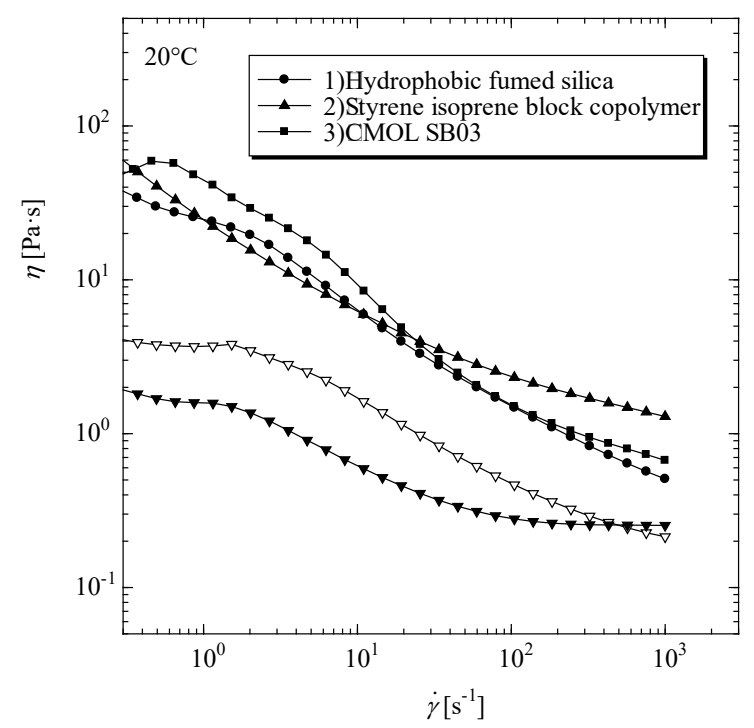

Figure 5. Steady flow characteristics of MRFs prepared with different stabilizing additives

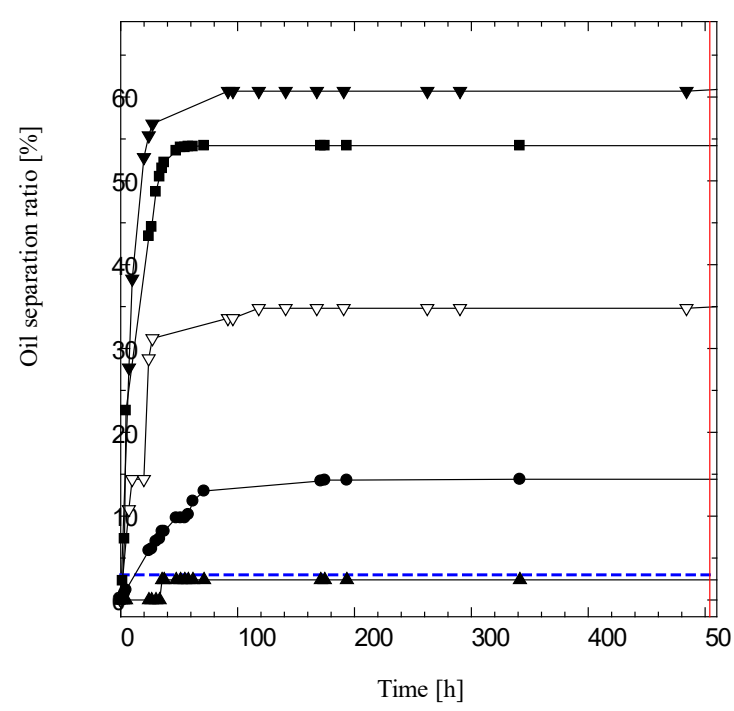

Figure 6. Oil separation ratio of MRFs prepared with different stabilizing additives. The test was conducted after 27 days to ensure enough mixing

After the stability test, the rheological properties (steady flow characteristics and viscoelasticity) of 
collected oils from the oil layers were conducted. From the results, we found that the rheological properties of oils collected from MRF with fumed silica and that with CMOL SB03 were almost the same as that of the base oil. In contrast, the oil collected from the MRF with the copolymer showed higher viscosity and viscoelasticity than that of the base oil. We expected that fumed silica and CMOL SB03 may exist on to the surface of the iron particles, while the copolymer molecules exist homogeneously in the oil. Therefore, the improvement of stability by the copolymer might cause from the increase of the viscosity and viscoelasticity of the solvent which can prevent the sedimentation of the particles. Meanwhile, fumed silica and CMOL SB03 may build certain structure or interaction for getting the particles involved; however, such structures were too weak to improve the stability of MRFs.

Figure 7 shows the TEM image of styrene isoprene block copolymer in the base oil. A certain specific functional group of the copolymer molecules may detected as many dots of similar distance in the image. Although the entwining of molecules cannot observed from the image, it might become the evidence that the molecules existed uniformly in the base oil.

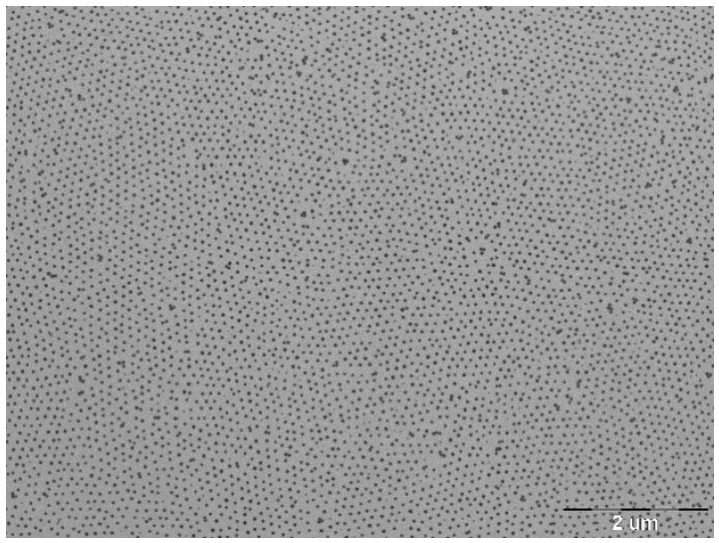

Figure 7. TEM image of styrene isoprene block copolymer in the base oil

Figure 8 shows magnetic characteristics for MRFs prepared with the three stabilizing additives. By comparing the linear line of shear stress, $\tau$, vs $B$ until 1.2 of MRF with $1 \mathrm{wt} \%$ oleic acid (without stabilizing additives, $\boldsymbol{\nabla}$ ), the strength of stresses for all the MRF increased with the addition of the stabilizing additives. Here, if the slope of the linear line is large, the MRF shows high performance. The order of the magnitude of the slopes for these stabilizing additives were fumed silica, copolymer, and CMOL SB03. We expected that highly-concentrated addition of the fumed silica compared to other two additives may relate to the highest strength of magnetic characteristic.

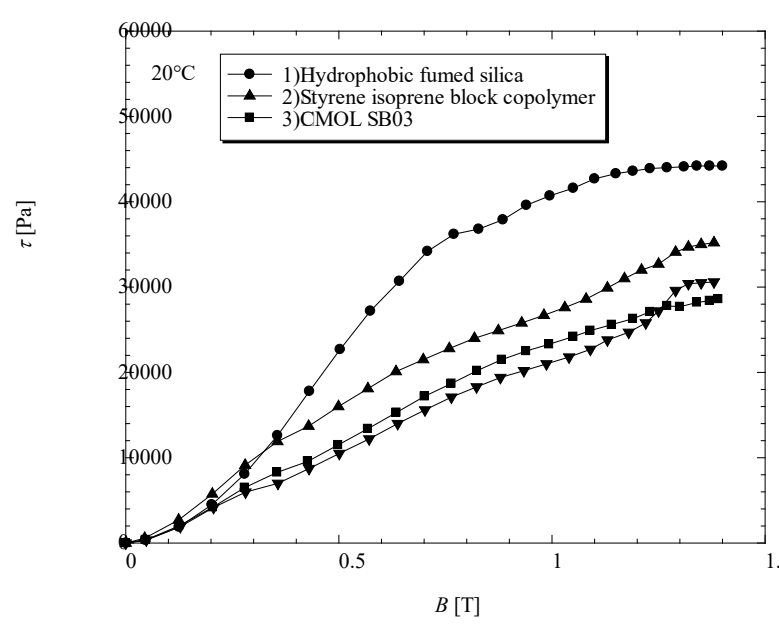

Figure 8. Magnetic characteristics for MRFs prepared with different stabilizing additives.

\section{Conclusion}

Suitable stabilizing additive for MRF should increase the stability without significant increase of viscosity, as well as increase the magnetic property. Three stabilizing additives were selected in this study and their rheological properties, stabilization ability for MRF, and the effect on the magnetic characteristics of MRF were evaluated.

\section{References}

Fang, F. F., Y. D. Liu, and H. J. Choi; "Carbon Nanotube Coated Magnetic Carbonyl Iron Microspheres Prepared by Solvent Casting Method and Their Magnetoresponsive Characteristics," Colloids Surf. A. Physicochem. Eng. Asp. 412 (2012) 47-56

Kim, M. S., Y. D. Liu, B. J. Park, C-Y. You, and H. J. Choi "Carbonyl Iron Particles Dispersed in a Polymer Solution and Their Rheological Characteristics under Applied Magnetic Field," J. Ind. Eng. Chem., 18, 664667 (2012)

Park, B. J., K. H. Song, and H. J. Choi; "Magnetic Carbonyl Iron Nanoparticle Based Magnetorheological Suspension and Its Characteristics," Mater. Lett., 63, 1350-1352 (2009)

Rabinow, J.; "The Magnetic Fluid Clutch," J. AIEE Trans., 67, 1308-1315 (1948)

Sakanishi, Y., Y. Narusaka, M. Itoh, T. Arita, and T. Saeki; "Development of New Series of Organogelators: N,N',N',N','-1,2,4,5-tetra Alkyl/Alkenyl Pyromellitamides," Nihon Reoroji Gakkaishi, 43, 1-9 (2015)

Usui, H., T. Saeki, and Y. Sano; "Stability Evaluation of Coal-Water Mixtures by Internal Structural Stress," $J$. Chem. Eng. Japan, 21, 602-607(1988) 